() Н.Ю. Свириденко*, Е.Г. Бессмертная, И.М. Беловалова, А.А. Михеенков, М.С. Шеремета, Л.В. Никанкина, Н.М. Малышева

Научный медицинский исследовательский центр эндокринологии, Москва, Россия

ОБОСНОВАНИЕ. По современным представлениям, эндокринная офтальмопатия (ЭОП) - это аутоиммунное заболевание, тесно связанное с аутоиммунной патологией щитовидной железы (ЩЖ). Ключевую роль в иммунопатогенезе аутоиммунных заболеваний ЩЖ, в том числе и ЭОП, играют цитокинопосредованные механизмы. Исследование цитокинового профиля, а также антител к тканевым антигенам имеет принципиальное значение для объяснения патогенеза ЭОП и будущих терапевтических стратегий.

ЦЕЛЬ. Исследование уровня цитокинов, аутоантител, иммуноглобулинов IgG и IgG4 как медиаторов аутоиммунного воспаления у пациентов с ЭОП и болезнью Грейвса (БГ).

МЕтоДЫ. В исследование были включены 52 пациента (104 орбиты) в активной фазе ЭОП и БГ, верифицированные по международным стандартам диагностики, не получавшие ранее лечения по поводу ЭОП. В контрольную группу были включены 14 человек (28 орбит), не имеющих аутоиммунной патологии. Определены концентрации иммуноглобулинов (IgG, IgG4), цитокинов (TNFa, IL-1 a, IL-1ß, IL-2, IL-4, IL-6, IL-8, IL-10, IL-17A, IL-13, TGF- $\beta 1$, TGF- $\beta 3$ ), растворимых рецепторов (sTNFa-RI и TNFa-R2, sIL-6R, IL-2R, антител к рецептору тиреотропного гормона (рТTГ), свободных фракций тироксина (T4) и трийодтиронина (Т3) - свТ4, свТ3, ТТГ) в сыворотке крови. Выполнено ультразвуковое исследование (УЗИ) ЩЖ, мультиспиральная компьютерная томография (МСКТ)/магнитно-резонансная томография (МРТ) орбит.

РЕЗУЛЬТАТЫ. Продолжительность ЭОП до поступления в отделение в среднем составила 8,8 $\pm 1,5$ мес и варьировала от 1 до 48 мес. По степени компенсации тиреотоксикоза находились в состоянии эутиреоза 24 пациента: ТТГ 3,3土0,7,

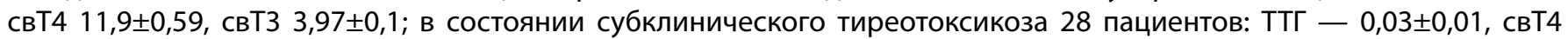

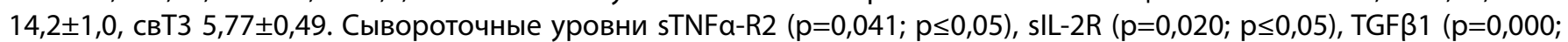
$\mathrm{p} \leq 0,001)$ были значимо выше у лиц с ЭОП по сравнению с контрольной группой. С увеличением длительности течения ЭОП повышались концентрации sTNFRa2 ( $p=0,038 ; p \leq 0,05)$ и TGF $\beta 1(p=0,011 ; p \leq 0,05)$, с увеличением тяжести концентрация sIL-6R ( $p=0,034 ; p \leq 0,05)$, с увеличением активности - sTNFa-R 1 ( $p=0,012 ; p \leq 0,05)$. Выявлено повышенное содержание $\lg 4$ в $\operatorname{lgG}$ (более $5 \%$ ) у $54 \%$ пациентов.

ЗАКЛючЕНИЕ. Высокие уровни солюбилизированных рецепторов цитокинов: sTNFa-R2, sIL-2R и цитокина TGF 1 у пациентов с длительно существующей нелеченой ЭОП и БГ в состоянии эутиреоза и субклинического тиреотоксикоза свидетельствуют об активации регуляторного звена иммунной системы, направленной на супрессию аутоиммунного процесса. Высокое содержание IgG4 в IgG и цитокина TGF $\beta 1$ может определять развитие фиброзных изменений в орбите. Снижение концентрации цитокина TGFß1 может свидетельствовать о неблагоприятном течении ЭОП.

КЛЮЧЕВЫЕ СЛОВА: болезнь Грейвса; эндокринная офтальмопатия; цитокины; аутоантитела; иммуноглобулины.

\title{
AUTOANTIBODIES, IMMUNOGLOBULINS AND CYTOKINE PROFILE IN PATIENTS WITH GRAVES' DISEASE AND GRAVES' ORBITOPATHY
}

(c) Natalya Yu. Sviridenko*, Elena G. Bessmertnaya, Irina M. Belovalova, Alexander A. Mikheenkov, Marina S. Sheremeta, Larisa V. Nikankina, Natalia M. Malysheva

Endocrinology Research Centre, Moscow, Russia

BACKGROUND: Graves' Orbitopathy (GO) - also known as Thyroid Eye Disease (TED) — is an autoimmune condition in the modern sense. It is closely associated with autoimmune thyroid diseases. Cytokine-mediated mechanisms play a critical part in immunopathogenesis of autoimmune thyroid diseases including GO. Investigating cytokine profiles as well as antibodies to tissue-specific antigens is essential for explaining GO pathogenesis and developing future therapeutic strategies.

AIMS: The study examines serum levels of cytokines, autoantibodies and immunoglobulins lgG and lgG4 as mediators of autoimmune inflammation in patients with GO and Graves' Disease (GD).

MATERIALS AND METHODS: The study included 52 patients (104 orbits) aged $25-70$ years (mean age 48,8 $\pm 12,3$ ) in the active phase of GO and GD verified with the international diagnostic standards. These patients did not get any treatment for GO before. The control group consisted of 14 individuals (28 orbits) aged 30-68 years without known autoimmune disease. 
Serum levels of IgG, IgG4, TNFa, IL-1 a, IL-1 $\beta$, IL-2, IL-4, IL-6, IL-8, IL-10, IL-17A, IL-13, sIL-6R, sTNFa- RI и TNFa- R2 IL-2R, TGF $\beta 1$, TGF $\beta 3$, antibodies to TSH-receptor, free T4, free T3 and TSH were measured. A diagnostic ultrasound exam of thyroid gland, multislice computed tomography (MSCT) / magnetic resonance imaging (MRI) of orbits were performed.

RESULTS: Mean duration of GO prior to being admitted to the centre was $8,8 \pm 1,5$ months (range: 1 - 48 months). According to the degree of thyrotoxicosis compensation: 24 patients were clinically euthyroid, TSH 3,3 $\pm 0,7 \mathrm{mU} / \mathrm{L}, \mathrm{free} \mathrm{T} 4$ $11,9 \pm 0,59 \mathrm{pmol} / \mathrm{L}$, free T3 3,97 $\pm 0,1 \mathrm{pmol} / \mathrm{L} ; 28$ patients were considered to have subclinical thyrotoxicosis: TSH 0,03 $\pm 0,01 \mathrm{mU} / \mathrm{L}$, free T4 14,2 $\pm 1,0 \mathrm{pmol} / \mathrm{L}$, free T3 5,77 $\pm 0,49 \mathrm{pmol} / \mathrm{L}$. Serum levels of sTNFa-R2 ( $p=0,041, p \leq 0,05), \operatorname{slL}-2 R(p=0,020, p \leq 0,05), T G F \beta 1$ $(p=0,000, p \leq 0,001)$ were significantly higher in patients with GO compared to the control group. Serum levels of sTNFRa2 $(p=0,038, p \leq 0,05)$ and TGF $\beta 1(P=0,011, p \leq 0,05)$ were positively correlated with the duration of GO. The positive correlations between the serum level of sIL-6R $(p=0,034, p \leq 0,05)$ and the severity of $G O$ as well as between the serum level of sTNFa- $R 1$ $(P=0,012, p \leq 0,05)$ and activity of $G O$ were observed. $54 \%$ of patients had elevated concentration level of $\lg G 4$ in $\lg G(>5 \%)$.

CONCLUSION: High levels of soluble cytokine receptors STNFa-R2 and SIL-2R and cytokine TGF $\beta 1$ in patients with long-standing untreated GO and GD being euthyroid or having subclinical thyrotoxicosis indicate activation of regulatory T cells aimed at suppressing autoimmune processes. High concentration level of lgG4 in lgG and cytokine TGF $\beta 1$ can determine the development of fibrotic changes in the orbital tissues. A decrease in the concentration of cytokine TGF $\beta 1$ can indicate an unfavorable course of the disease GO.

KEYWORDS: Graves' disease; Graves' orbitopathy; cytokines; autoantibodies; immunoglobulins.

\section{АКТУАЛЬНОСТЬ}

Болезнь Грейвса (БГ) является органоспецифическим аутоиммунным заболеванием. Примерно у половины пациентов с БГ, а по данным визуализирующих методов исследования (мультиспиральная компьютерная томография (МСКТ), магнитно-резонансная томография (MPT)), - в 80\% [1, 2] развивается аутоиммунное поражение тканей орбиты - эндокринная офтальмопатия (ЭОП). По современным представлениям, главную роль в патогенезе БГ и ЭОП играют аутореактивные Т-лимфоциты, реагирующие с общими для щитовидной железы (ЩЖ) и тканей орбиты антигенами [3]. Т-лимфоциты вызывают каскад патологических реакций, приводящих к образованию антител к рецептору тиреотропного гормона (рТТГ) и рецептору инсулиноподобного ростового фактора (IGF-1), а также иммуноглобулинов lgG4, возможно, определяющих вовлечение различных анатомических структур глаза в аутоиммунный процесс $[4,5]$. Существующие терапевтические подходы к лечению ЭОП основываются на неспецифической иммуносупрессии глюкокортикоидами (ГК) и лучевой терапии орбит [6, 7]. Среди других возможных терапевтических подходов рассматриваются: ингибиторы цитокинов и их рецепторов, ингибиторы клеточной пролиферации Т- и В-лимфоцитов [8-10]. Исследование цитокинового профиля, а также антител к тканевым антигенам имеет принципиальное значение для объяснения патогенеза ЭОП и будущих терапевтических стратегий.

Цель - исследование цитокинов, аутоантител, иммуноглобулинов IgG и IgG4 как медиаторов аутоиммунного воспаления у пациентов с ЭОП и БГ.

\section{МЕТОДЫ}

Дизайн исследования

Проведено обсервационное одноцентровое одномоментное выборочное контролируемое исследование.

Критерии соответствия

В исследование были включены пациенты с БГ и ЭОП, верифицированными по международным стандартам диагностики [11]. Объектом исследования являлся пациент и его глаза. Учитывая то, что у одного и того же пациента глаза имеют разную степень выраженности клинических симптомов ЭОП, обработка результатов исследования проводилась отдельно для каждого глаза (шкала клинической активности - CAS) и каждой орбиты (МСКТ). Тяжесть и активность ЭОП оценивались по наиболее пораженному глазу. Критериями исключения были пациенты, получавшие ранее системную глюкокортикоидную или лучевую терапию орбит, имевшие сопутствующие аутоиммунные заболевания, сопутствующие хронические инфекционные заболевания, перенесенные вирусные заболевания в течение последних 3 мес, беременные и кормящие женщины. В контрольную группу были включены лица, не имеющие заболеваний ЩЖ, глаз и аутоиммунной патологии.

\section{Условия проведения}

Исследование проводилось в ФГБУ «НМИЦ эндокринологии» Минздрава РФ в отделе терапевтической эндокринологии.

\section{Продолжительность исследования}

Включение пациентов в исследование проведено в 2018-2019 гг.

\section{Описание медицинского вмешательства}

Всем пациентам проводились общеклиническое обследование со сбором анамнеза, физикальное обследование, определение уровня ТТГ, свободных фракций тироксина (Т4) и трийодтиронина (Т3), антител к рТТГ, ультразвуковое исследование (УЗИ) щитовидной железы (ЩЖ) с применением цветовой допплерографии. Всем пациентам и группе контроля было выполнено стандартное офтальмологическое исследование на базе отделения диабетической ретинопатии и офтальмохирургии ФГБУ «НМИЦ эндокринологии» Минздрава России (ЭНЦ) (зав. отд. д.м.Н. Липатов Д.В.): визометрия, определение уровня внутриглазного давления, биомикроскопия переднего отдела глаза, экзофтальмометрия, компьютерная периметрия, биомикроскопия 
хрусталика и стекловидного тела с помощью щелевой лампы, обратная и прямая офтальмоскопия, визуализация орбит - МСКТ, МРТ. Диагноз ЭОП верифицировали соответственно рекомендациям EUGOGO [12]. Тяжесть ЭОП оценивалась по классификации NOSPECS [13]. Активность ЭОП оценивалась по шкале клинической активности CAS [14].

\section{Основной исход исследования}

Проводилось определение антител к рТТГ, иммуноглобулинов IgG и IgG4 (за исключением группы контроля), цитокинов и их растворимых рецепторов.

\section{Анализ в подгруппах}

Для проведения исследования были сформированы группы по активности, тяжести, длительности ЭОП, степени компенсации тиреотоксикоза

\section{Методы регистрации исходов}

Содержание IgG4, фактора некроза опухоли альфа (TNFa), интерлейкинов (IL-1a, IL-1 $\beta$, IL-2, IL-4, IL-6, IL-8, IL-10, IL-17A, IL-13), растворимых рецепторов (sIL-6R), трансформирующего ростового фактора бета 1 (TGF $\beta 1$ ) в образцах сыворотки определяли с помощью коммерческих наборов фирмы BenderMedSystems GmbH (Австрия), sTNFa-RI и TNFa-R2 - наборами R\&Dsystems (США-Канада), IgG - набором Seramun Diagnostica GmbH (Германия), IL-2R - набором RayBiotech (США), TGF 33 - набором Cloud-Clone Corp. (Китай). Все вышеуказанные исследования выполняли методом иммуноферментного анализа, измерение оптической плотности проводили на счетчике 1420 Multilabel Counter VICTOR2 (Perkin Elmer). Оценку функции ЩЖ - уровень ТТГ, свТ3, свТ4 - определяли методом усиленной хемилюминесценции на автоматическом анализаторе Architect (Abbott Diagnostics, США). Референсные значения для базального ТТГ 0,25-3,5 мМЕ/л, свТ4 - 9,0-20,0 пмоль/л, свТ3 - 2,5-5,5 пмоль/л. Антитела к рТТГ (референсные значения 0,00-1,75 ME/л) определяли методом электрохемилюминесцентного анализа на иммунохимическом автоматическом анализаторе Cobas 6000 (Roche Diagnostics, Германия). Забор крови проводился утром натощак, после сна в условиях стационара. Лабораторные исследования проводили на базе клинико-диагностической лаборатории ФГБУ «НМИЦ эндокринологии» МЗ РФ (зав. лаб. к.м.н. Никанкина Л.В.).

УзИ ЩЖ выполнено на ультразвуковом сканеpe Toshiba Aplio 790 датчиком переменной частоты 7,5-10 МГц с применением цветовой допплерографии на базе отделения ультразвуковой диагностики ЭНЦ (зав. к.м.н. Солдатова Т.В.). МРТ, МСКТ орбит проводились в отделе лучевой диагностики НМИЦ (зав. д.М.н. Воронцов А.В.) на 320-срезовом аппарате Aquilion One (Toshiba, Япония) со сканированием в автоматическом режиме. Принимая во внимание возможность разной выраженности патологического процесса в обеих орбитах, каждую орбиту оценивали отдельно в 3 проекциях: аксиальной, корональной, сагиттальной. Определение минимальных и максимальных значений плотности экстраокулярных мышц (ЭОМ) и ретробульбарной клетчатки (РБК) проводили в корональной и аксиальной проекциях, отступив 1-2 мм от контуров мышцы [15].

\section{Этическая экспертиза}

Работа одобрена локальным Этическим комитетом при ФГБУ «НМИЦ эндокринологии» Минздрава РФ. Протокол заседания локального этического комитета № 17 от 27.09.2017 г.

\section{Статистический анализ}

Статистическая обработка данныхвыполнена на персональном компьютере с использованием пакета программ статистического анализа данных Statistica 6.13 (StatSoft Inc., США) и приложения Microsoft Excel for Windows. Для количественных признаков рассчитывались: средние, минимальные и максимальные значения, стандартные отклонения (полученные результаты представлены в виде $\mathrm{M} \pm \mathrm{SD}$, где $\mathrm{M}$ - среднее арифметическое значение, SD стандартное отклонение) либо медиана и квартили - Ме [25; 75]. Анализ межгрупповых различий при нормальном распределении признака проводился с использованием t-критерия Стьюдента. Для сравнения независимых выборок при распределении признака, отличавшегося от нормального, использовался критерий Манна-Уитни. Критический уровень значимости при проверке статистических гипотез принимался равным 0,05 $(p<0,05)$.

\section{РЕЗУЛЬТАТЫ}

\section{Участники исследования}

В исследование были включены 52 пациента (104 орбиты) в активной фазе ЭОП. Средний возраст составил $48,8 \pm 12,3$ года в диапазоне от 25 до 70 лет, 38 женщин, 14 мужчин. В контрольную группу были включены 14 человек (28 орбит), в возрасте от 30 до 68 лет, не имеющих аутоиммунной патологии.

Хронологический анализ показал, что у 30,8\% пациентов симптомы ЭОП и БГ развились одновременно, у 11,5\% - ЭОП манифестировала первой, до появления симптомов тиреотоксикоза, у 57,7\% - первой манифестировала БГ, промежуток до появления симптомов ЭОП варьировал от 2 до 72 мес. Продолжительность ЭОП до поступления в отделение в среднем составила 8,8ะ1,5 мес и варьировала от 1 до 48 мес. До поступления в отделение пациенты уже принимали тиреостатики, но не лечились по поводу ЭОП. Длительность лечения ти-

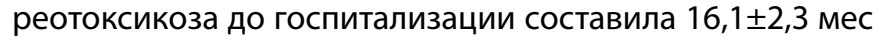
и варьировала от 1 до 60 мес.

Клинико-инструментальная характеристика пациентов с ЭОП представлена в табл. 1. Все пациенты были консультированы офтальмологом. Согласно шкале клинической активности CAS, пациенты имели активную фазу ЭОП, средний балл составил $4 \pm 0,18$. По степени активности пациенты были разделены на 2 группы: с низкой активностью CAS - 3-4 (73\%) и высокой активностью CAS - 5-7 (27\%).

По степени компенсации тиреотоксикоза пациенты были разделены на 2 группы: в состоянии эутиреоза

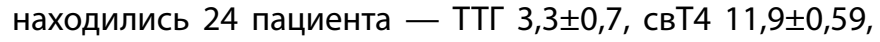
свТ3 $3,97 \pm 0,1$; в состоянии субклинического тиреоток-

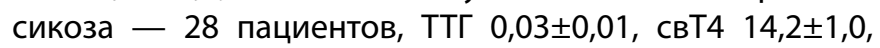

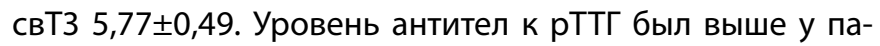
циентов с субклиническим тиреотоксикозом - 17,9 $\pm 3,4$

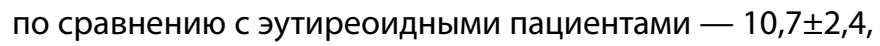
$\mathrm{p}<0,1$, но не достигал статистической значимости. 
Таблица 1. Клиническая характеристика пациентов с эндокринной офтальмопатией

\begin{tabular}{|c|c|c|c|}
\hline & \multicolumn{2}{|c|}{ Шкала клинической активности } & \multirow{2}{*}{$\begin{array}{c}\text { Различия между } \\
\text { группами } \\
\mathbf{p} \\
\end{array}$} \\
\hline & CAS 3-4 & CAS 5-7 & \\
\hline Всего глаз, n & 60 & 34 & \\
\hline 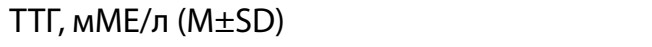 & $1,02 \pm 0,27$ & $1,2 \pm 0,5$ & н/д \\
\hline Субклинический тиреотоксикоз/эутиреоз & $53,8 \% / 46,2 \%$ & $50 \% / 50 \%$ & \\
\hline свT4, пмоль/л (M $\pm S D)$ & $13,1 \pm 0,9$ & $13,9 \pm 0,9$ & н/д \\
\hline свT3, пмоль/л (M $\pm S D)$ & $4,8 \pm 0,5$ & $5,1 \pm 0,3$ & н/д \\
\hline $\begin{array}{l}\text { Антитела к рТТГ, ME/л } \\
\text { Медиана }[25 ; 75]\end{array}$ & $7,5[1,77 ; 17,47]$ & $18,6[8,99 ; 40]$ & $\begin{array}{l}p=0,020 \\
p \leq 0,05\end{array}$ \\
\hline Диплопия & $46,6 \%$ & $71 \%$ & \\
\hline Косоглазие & $26,6 \%$ & $29 \%$ & \\
\hline Экзофтальм по Гертелю, мм (M士SD) & $19,8 \pm 0,6$ & $24,2 \pm 1,1$ & $p=0,000, p \leq 0,001$ \\
\hline 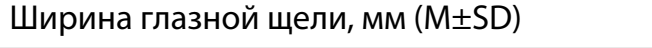 & $10,5 \pm 0,4$ & $11,3 \pm 0,8$ & $p=0,300, н / д$ \\
\hline Лагофтальм (M⿻SD) & $20 \%(1,6 \pm 0,4 \mathrm{Mm})$ & $50 \%(2,1 \pm 0,38 \mathrm{мм})$ & \\
\hline Хемоз & $70 \%$ & $100 \%$ & \\
\hline 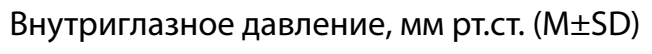 & $18,9 \pm 0,9$ & $24,0 \pm 1,8$ & $p=0,007, p \leq 0,01$ \\
\hline Оптическая нейропатия & $13,3 \%$ & $71,4 \%$ & \\
\hline Тяжесть средняя/тяжелая & $83 \% / 17 \%$ & $29 \% / 71 \%$ & \\
\hline
\end{tabular}

Таблица 2. Цитокиновый профиль у пациентов с эндокринной офтальмопатией и контрольной группы.

\begin{tabular}{|c|c|c|c|}
\hline & $\begin{array}{c}\text { Пациенты ЭОП } \\
\text { CAS 3-7 }\end{array}$ & Контроль & $\begin{array}{c}\text { Различия между } \\
\text { группами }\end{array}$ \\
\hline TGF $\beta 1$, пг/мл & 20049,9 & 14320,7 & $p=0,0001$ \\
\hline Медиана [25; 75] & {$[16570,5 ; 30734,9]$} & {$[11058,7 ; 15$ 315,7] } & $\mathrm{p}=0,000$ \\
\hline $\mathrm{M} \pm \mathrm{m}$ & $24450,4 \pm 1772,2$ & $12646,8 \pm 1335,9$ & $\mathrm{p} \leq 0,001$ \\
\hline sTNFa-R1, пг/мл & 1540,6 & 1713,5 & $p=0,096$ \\
\hline Медиана [25; 75] & {$[1022,4 ; 1731,3]$} & {$[1416,9 ; 2092,5]$} & H/д \\
\hline sTNFa-R2, пг/мл & 2889,5 & 2346,7 & $p=0,041$ \\
\hline Медиана [25; 75] & {$[2187,4 ; 3497,1]$} & {$[2065,1 ; 2730,9]$} & $\mathrm{p} \leq 0,05$ \\
\hline sIL-2R, пг/мл & 146,2 & 79,7 & $p=0,020$ \\
\hline Медиана [25; 75] & {$[94,7 ; 246,5]$} & {$[52,1 ; 102,8]$} & $p \leq 0,05$ \\
\hline sIL-6R, пг/мл & 127 & 104,1 & $p=0,138$ \\
\hline Медиана [25; 75] & {$[99,5 ; 162,6]$} & {$[85,5 ; 148]$} & н/д \\
\hline IL-8, пг/мл & 12,1 & 10,9 & $p=0,061$ \\
\hline Медиана [25; 75] & $8,5 ; 17,6]$ & {$[7,7 ; 17,6]$} & $p=0,568$ \\
\hline$M \pm m$ & $16,8 \pm 6,4$ & $12,9 \pm 2,1$ & н/д \\
\hline \multirow{3}{*}{$\begin{array}{l}\text { Антитела к рТТГ (ME/л) } \\
(\mathrm{M} \pm \mathrm{SD}) \\
\text { Медиана }[25 ; 75]\end{array}$} & & \multirow{3}{*}{-} & \\
\hline & $14,0 \pm 2,1$ & & \\
\hline & $9,16[2,7 ; 18,3]$ & & \\
\hline
\end{tabular}

\section{ОСНОВНЫЕ РЕЗУЛЬТАТЫ ИССЛЕДОВАНИЯ}

Исследование цитокинового профиля

Сывороточные уровни TNFa, IL-1a, IL-1 $\beta$, IL-2, IL-4, IL-6, IL-10, IL-17A, IL-13, за исключением IL-8, определялись в следовых количествах и у пациентов с ЭОП и в контрольной группе в дальнейшем не анализировались. В отличие от вышеуказанных цитокинов сывороточные уровни растворимых рецепторов цитокинов sTNFa-R1, sTNFa-R2, sIL-R2, sIL-R6 и уровни IL-8 и TGF $\beta 1$ определялись в высоких концентрациях. Результаты представлены в таблице 2.

При сравнении пациентов в активной фазе ЭОП (CAS 3-7) с контрольной группой были выявлены более высокие, статистически значимые различия показателей цитокина TGF $\beta 1(p=0,0001)$, рецепторов цитокинов sTNFa-R2 ( $p=0,041, p \leq 0,05)$, sIL-2R ( $p=0,020, p \leq 0,05)$. 
При сравнении пациентов с разным уровнем активности (CAS 3-4 и CAS 5-7) достоверные различия выявлены только для sTNFa-R1 ( $P=0,012, \mathrm{p} \leq 0,05)$ и антител к рTTГ ( $p=0,020, p \leq 0,05)$ (табл. 3). Концентрации TGF $\beta 1$ и SIL-2R имели тенденцию к снижению у пациентов с более высокой активностью ЭОП, но не достигали статистической значимости.
При сравнении пациентов с разной степенью тяжести (средней и тяжелой) достоверные различия выявлены только для sIL-6R ( $p=0,034 ; p \leq 0,05)$.

У пациентов с субклиническим тиреотоксикозом и эутиреозом статистически значимых различий в уровне рецепторов цитокинов не выявлено (табл. 4), за исключением sIL-2R ( $p=0,024)$.

Таблица 3. Цитокиновый профиль у пациентов с разной степенью активности эндокринной офтальмопатии.

\begin{tabular}{|c|c|c|c|}
\hline & $\begin{array}{c}\text { Пациенты } \\
\text { CAS 3-4 }\end{array}$ & $\begin{array}{c}\text { Пациенты } \\
\text { CAS 5-7 }\end{array}$ & $\begin{array}{c}\text { Различия между } \\
\text { группами }\end{array}$ \\
\hline TGF $\beta 1$, пг/мл & 25093,2 & 19733,5 & $p=0,199$ \\
\hline Медиана [25; 75] & {$[17416,2 ; 34031,6]$} & {$[14516,6 ; 23742,3]$} & н/д \\
\hline sTNFa-R1, пг/мл & 1233,3 & 1654,5 & $p=0,012$ \\
\hline Медиана [25; 75] & {$[972,5 ; 1733,6]$} & {$[1594,0 ; 2126,7]$} & $p \leq 0,05$ \\
\hline sTNFa-R2, пг/мл & 2947,1 & 2556,1 & $p=0,492$ \\
\hline Медиана [25; 75] & {$[2439,4 ; 3369,7]$} & {$[2109,5 ; 3569,3]$} & н/д \\
\hline sIL-2R, пг/мл & 174,2 & 99,8 & $p=0,056$ \\
\hline Медиана [25; 75] & {$[102,9 ; 276,2]$} & {$[65,5 ; 192,9]$} & Н/д \\
\hline sIL-6R, пг/мл & 127,0 & 104,1 & $p=0,139$ \\
\hline Медиана [25; 75] & {$[99,5 ; 162,6]$} & {$[85,5 ; 148]$} & н/д \\
\hline IL-8, пг/мл & 5,5 & 10,9 & $p=0,089$ \\
\hline Медиана [25; 75] & {$[3,2 ; 7,7]$} & {$[5,9 ; 13,2]$} & н/д \\
\hline Антитела к рТТГ, МЕ/л & 7,5 & 18,6 & $p=0,020$ \\
\hline Медиана [25; 75] & {$[1,77 ; 17,47]$} & {$[8,99 ; 40]$} & $p \leq 0,05$ \\
\hline
\end{tabular}

Таблица 4. Цитокиновый профиль у пациентов с субклиническим тиреотоксикозом и эутиреозом.

\begin{tabular}{|c|c|c|c|}
\hline \multirow[t]{2}{*}{ Показатели } & $\begin{array}{c}\text { Пациенты, } \\
\text { субклинический } \\
\text { тиреотоксикоз }\end{array}$ & $\begin{array}{l}\text { Пациенты, } \\
\text { эутиреоз }\end{array}$ & $\begin{array}{c}\text { Различия между } \\
\text { группами }\end{array}$ \\
\hline & $M \pm m$ & $M \pm m$ & $\mathbf{p}$ \\
\hline 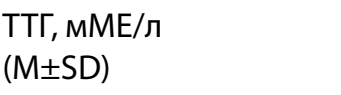 & $0,03 \pm 0,01$ & $3,3 \pm 0,7$ & $\begin{array}{l}p=0,000 \\
p \leq 0,01\end{array}$ \\
\hline $\begin{array}{l}\text { свТ4, пмоль/л } \\
(\mathrm{M} \pm \mathrm{SD})\end{array}$ & $14,2 \pm 1,0$ & $11,9 \pm 0,59$ & $\begin{array}{l}p=0,060 \\
p \leq 0,01\end{array}$ \\
\hline $\begin{array}{l}\text { свТ3, пмоль/л } \\
(\mathrm{M} \pm \mathrm{SD})\end{array}$ & $5,77 \pm 0,49$ & $3,97 \pm 0,1$ & $\begin{array}{l}p=0,003 \\
p \leq 0,01\end{array}$ \\
\hline $\begin{array}{l}\text { Антитела к рТТГ, ME/л } \\
(\mathrm{M} \pm \mathrm{SD})\end{array}$ & $17,9 \pm 3,4$ & $10,7 \pm 2,4$ & $\begin{array}{l}p=0,085 \\
p \leq 0,1\end{array}$ \\
\hline TGF $\beta 1$, пг/мл & 22660,1 & 19442,0 & $p=0,166$ \\
\hline Медиана [25; 75] & {$[17416,2 ; 35592,7]$} & {$[14983,8 ; 25465,7]$} & н/д \\
\hline sTNFa-R1, пг/мл & 1593,2 & 1558,2 & $p=0,270$ \\
\hline Медиана [25; 75] & {$[1172,9 ; 1946,2]$} & {$[884,9 ; 1816,5]$} & н/д \\
\hline sTNFa-R2, пг/мл & 3069,9 & 2581,0 & $p=0,177$ \\
\hline Медиана [25; 75] & {$[2646,3 ; 3654,5]$} & {$[2019,7 ; 3359,8]$} & н/д \\
\hline sIL-2R, пг/мл & 225,1 & 107,0 & $p=0,024$ \\
\hline Медиана [25; 75] & {$[113,4 ; 290,6]$} & {$[75,1 ; 182,7]$} & $\mathrm{p} \leq 0,05$ \\
\hline sIL-6R, пг/мл & 128,8 & 126,5 & $p=0,294$ \\
\hline Медиана [25; 75] & {$[107,4 ; 172,1]$} & {$[93,8 ; 153,0]$} & н/д \\
\hline IL-8, пг/мл & 5,0 & 8,5 & $p=0,260$ \\
\hline Медиана [25; 75] & {$[4,1 ; 10,9]$} & {$[5,9 ; 13,2]$} & н/д \\
\hline
\end{tabular}


Таблица 5. Цитокиновый профиль у пациентов с разной длительностью эндокринной офтальмопатии до начала ее лечения.

\begin{tabular}{|c|c|c|c|c|c|}
\hline & \multicolumn{3}{|c|}{$\begin{array}{c}\text { Длительность ЭОП } \\
\text { до начала ее лечения, мес }\end{array}$} & \multicolumn{2}{|c|}{$\begin{array}{c}\text { Различия } \\
\text { между группами }\end{array}$} \\
\hline & $\begin{array}{c}1-4 \text { мec } \\
1 \\
\text { Me }[25 ; 75]\end{array}$ & $\begin{array}{c}5-9 \text { мeс } \\
2 \\
\text { Me }[25 ; 75]\end{array}$ & $\begin{array}{c}\geq 10 \text { мeс } \\
3 \\
\text { Me }[25 ; 75]\end{array}$ & $p \leq 1,2$ & $p \leq 1,3$ \\
\hline TGFß1, пг/мл & 19134,9 & 25685,0 & 27171,0 & $p=0,048$ & $p=0,041$ \\
\hline Медиана [25; 75] & [12 320; 19 975] & [17 708; 31750$]$ & [16 997; 42 759] & $p \leq 0,05$ & $p \leq 0,05$ \\
\hline $\begin{array}{l}\text { sTNFa-R1, пг/мл } \\
\text { Медиана [25; 75] }\end{array}$ & $\begin{array}{c}16343 \\
{[1040,5 ; 1816,5]}\end{array}$ & $\begin{array}{c}1566,2 \\
{[1019,2 ; 1720]}\end{array}$ & $\begin{array}{c}1522 \\
{[1051,5 ; 1841]}\end{array}$ & $\begin{array}{l}\mathrm{p}=0,510 \\
\text { н/д }\end{array}$ & $\begin{array}{l}p=0,746 \\
\text { н/д }\end{array}$ \\
\hline $\begin{array}{l}\text { sTNFa-R2, пг/мл } \\
\text { Медиана [25; 75] }\end{array}$ & $\begin{array}{c}2462,3 \\
{[1979 ; 3369,7]}\end{array}$ & $\begin{array}{c}2696,9 \\
{[2060 ; 2962]}\end{array}$ & $\begin{array}{c}3150,3 \\
{[2722,6 ; 4026]}\end{array}$ & $\begin{array}{l}\mathrm{p}=0,984 \\
\mathrm{H} / \mathrm{g}\end{array}$ & $\begin{array}{l}p=0,038 \\
p \leq 0,05\end{array}$ \\
\hline $\begin{array}{l}\text { slL-2R, пг/мл } \\
\text { Медиана [25; 75] }\end{array}$ & $\begin{array}{c}142,9 \\
{[98,8 ; 239,5]}\end{array}$ & $\begin{array}{c}165,6 \\
{[90,5 ; 300,2]}\end{array}$ & $\begin{array}{c}102,9 \\
{[73,4 ; 210,8]}\end{array}$ & $\begin{array}{l}\mathrm{p}=0,765 \\
\mathrm{H} / \mathrm{д}\end{array}$ & $\begin{array}{l}\mathrm{p}=0,516 \\
\mathrm{H} / \mathrm{g}\end{array}$ \\
\hline $\begin{array}{l}\text { slL-6R, пг/мл } \\
\text { Медиана [25; 75] }\end{array}$ & $\begin{array}{c}121,9 \\
{[100 ; 152,9]}\end{array}$ & $\begin{array}{c}110,8 \\
{[99,5 ; 145,3]}\end{array}$ & $\begin{array}{c}150,1 \\
{[126,8 ; 184]}\end{array}$ & $\begin{array}{l}\mathrm{p}=0,789 \\
\mathrm{H} / \mathrm{д}\end{array}$ & $\begin{array}{l}\mathrm{p}=0,108 \\
\mathrm{H} / \mathrm{g}\end{array}$ \\
\hline $\begin{array}{l}\text { IL-8, пг/мл } \\
\text { Медиана [25; 75] }\end{array}$ & $\begin{array}{c}7,7 \\
{[4,6 ; 12,1]}\end{array}$ & & $\begin{array}{c}7,9 \\
{[4,1 ; 17,6]}\end{array}$ & & $\begin{array}{l}\mathrm{p}=0,857 \\
\text { н/д }\end{array}$ \\
\hline $\begin{array}{l}\text { CAS, баллы } \\
(\mathrm{M} \pm \mathrm{SD})\end{array}$ & $4,0 \pm 0,3$ & $3,8 \pm 0,3$ & $4,1 \pm 0,1$ & & \\
\hline Диплопия & $33,3 \%$ & $68,8 \%$ & $77,8 \%$ & & \\
\hline Косоглазие & $5,5 \%$ & $43,8 \%$ & $44,4 \%$ & & \\
\hline
\end{tabular}

Таблица 6. Концентрации IgG и lgG4 у пациентов с эндокринной офтальмопатией.

\begin{tabular}{lcc}
\hline \multicolumn{1}{c}{ Показатели } & $\mathbf{M} \pm \mathbf{m}$ & \% отношение \\
\hline $\operatorname{lgG4,~мкг/мл~}$ & $754,3 \pm 146,6$ & $\begin{array}{c}15 \% \text { выше нормы } \\
>1350 \text { мкг/мл }\end{array}$ \\
\hline $\operatorname{lgG,~мкг/мл~}$ & $8849,6 \pm 382,6$ & \\
\hline $\begin{array}{l}\text { Oтношение } \\
\operatorname{lgG} 4 / \operatorname{lgG} \%\end{array}$ & & $54 \%>5 \%$ \\
\hline
\end{tabular}

Мы проанализировали цитокиновый профиль в зависимости от длительности ЭОП до начала ее лечения (табл. 5). Уровни TGF $\beta 1$ и TNFa-R2 статистически значимо повышались с увеличением длительности ЭОП до поступления в стационар и начала ее лечения ( $p=0,048 ; p=0,041$ и $\mathrm{p}=0,038$ соответственно). TNFa-R1 и IL-2R имели тенденцию к снижению, но статистически незначимую. Уровень IL-6R оставался без изменений. С увеличением длительности течения офтальмопатии нарастала частота диплопии и косоглазия.

Концентрация IgG4 превышала норму более 1350 мкг/мл у 15\% пациентов. Концентрация lgG4 превышала норму (более 5\% от общего IgG сыворотки крови) у 54\% пациентов и варьировала от 6,7 до 20,\% (табл. 6).

\section{ОБСУЖДЕНИЕ}

За последние десятилетия в связи с признанием аутоиммунной природы БГ достаточно подробно были изучены показатели В-клеточного иммунитета. Было доказано, что антитела к рТТГ играют ведущую роль не только в патогенезе БГ [16], но и являются прогностическими маркерами активности и тяжести ЭОП [17]. Реакции Т-клеточного иммунитета изучены в меньшей степени. Ключевую роль в иммунопатогенезе аутоиммунных заболеваний ЩЖ, в том числе и ЭОП, играют цитокинопосредованные механизмы [18]. Цитокины вырабатываются в основном Т-клетками иммунной системы, но могут секретироваться макрофагами, эндотелиальными клетками, орбитальными фибробластами и т.д. [19]. Цитокины - антиген-неспецифические факторы. Поэтому специфическая диагностика аутоиммунных заболеваний с помощью определения уровня цитокинов невозможна. Но определение их концентрации в крови дает информацию о тяжести воспалительного процесса и о прогнозе заболевания [20]. Поскольку цитокины при ЭОП образуются локально в пораженном органе, сывороточная концентрация может не отражать их уровень в соответствующих тканях. В нашем исследовании уровни TNFa, IL-1a, IL-1 $\beta$, IL-2, IL-4, IL-6, IL-10, IL-17A, IL-13 в сыворотке крови определялись в следовых количествах и у пациентов с ЭОП и в контрольной группе и в дальнейшем не анализировались.

Для проявления биологической активности цитокина необходимо присутствие на поверхности клеток специфических рецепторов [21]. Цитокин взаимодействует с внешней, экстрацеллюлярной, частью рецептора. Внеклеточный домен мембранного рецептора отщепляется и поступает в кровь - растворимый (солюбилизированный) рецептор. Главное биологическое значение солюбилизированных рецепторов состоит в блокировке эффектов соответствующих цитокинов. В нашем исследовании 
мы проанализировали концентрацию рецепторов цитокинов, которая значительно превышала концентрацию соответствующего цитокина.

TNFa - провоспалительный цитокин. Ранее было показано, что TNFa играет важную роль в аутоиммунном воспалительном процессе при ЭОП [22], в тканях орбиты рецепторы к TNFa определяются в большом количестве [23]. Его sTNFa-R1 экспрессируется клетками большинства тканей, a sTNFa-R2 экспрессируется более ограниченным образом в определенных популяциях лимфоцитов, включая Т-регуляторные клетки (Tregs) [24]. В нашем исследовании сывороточные уровни sTNFa-R2 были значимо выше у лиц с ЭОП по сравнению с контрольной группой $(p=0,041 ; p \leq 0,05)$. sTNFa-R2 стабилизирует циркулирующий TNFa и увеличивает период полураспада данного цитокина в сыворотке крови, являясь его «ловушкой». Его повышенная концентрация свидетельствует об активности иммунной системы, преимущественно Tregs, направленной на подавление аутоиммунного процесса. В нашем исследовании концентрация sTNFRa2 повышалась с увеличением длительности течения ЭОП ( $p=0,0387 ; p \leq 0,05)$, что свидетельствует об активации регуляторного звена иммунной системы с увеличением длительности ЭОП. sTNFa-R1 также стабилизирует циркулирующий TNFa и увеличивает период полураспада данного цитокина в сыворотке крови. Его основная функция - запустить апоптоз, т.е. гибель клетки [25]. В нашем исследовании уровни sTNFa-R1 не отличались от контроля, но значимо повышались у пациентов с более высокой активностью $(p=0,012 ; p \leq 0,05)$.

Орбитальные фибробласты продуцируют трансформирующий фактор роста бета (TGF $\beta 1)$, который стимулирует выработку гликозаминогликанов, а также дифференцировку орбитальных фибробластов в миофибробласты [26], что определяет развитие фиброза, особенно на поздних стадиях заболевания. В нашем исследовании уровень TGF $\beta 1$ был значимо выше у пациентов с ЭОП по сравнению со здоровыми пациентами $(p=0,0001)$ и повышался с увеличением длительности течения ЭОП ( $p=0,041, p \leq 0,05)$, что косвенно может свидетельствовать о прогрессировании фиброза в орбите. О прогрессировании фиброза также свидетельствует более высокая частота диплопии и косоглазия в отдаленные сроки течения ЭОП (табл. 5). Кроме того, TGF $\beta 1$ подавляет экспрессию рТТГ на фибробластах и супрессирует иммунный ответ [27].

Интерлейкин IL-2 продуцируется активированными T-хелперами (Th1). IL-2 оказывает пролиферирующее и активирующее воздействие на Т-лимфоциты (киллеры) и В-клетки, а также на натуральные киллеры и определяет длительность иммунного ответа [28]. IL-2 способствует генерации, выживаемости и функциональной активности Tregs Foxp3+ (Treg или T-супрессоры; Foxp3 - транскрипционный фактор, регулирующий транскрипцию генов, ответственных за дифференцировку Т-клеток и экспрессию цитокинов) [29]. В его отсутствие наблюдается глубокий дефицит Т-супрессоров, приводящий к развитию аутоиммунных заболеваний. Свою активность IL-2 проявляет после связывания со специфическим клеточным рецептором IL-2R. Определение уровня SIL-2R позволяет выявлять и контролировать активацию Treg как супрессоров аутоиммунного процесса. В нашем исследовании сывороточный уровень SIL-2R у пациентов с ЭОП был значимо выше контроля ( $p=0,020 ; p \leq 0,05)$. Уровень slL-2R снижался с длительностью ЭОП, но не имел достоверной значимости.

IL-6 является провоспалительным цитокином и повышается в острую воспалительную фазу инфекционных заболеваний. Он стимулирует дифференцировку В-лимфоцитов и активирует Т-лимфоциты с последующим снижением Treg [30, 31], приводя к потере аутотолерантности при аутоиммунных заболеваниях. По нашим данным, комплекс IL-6/sIL-6R не имел отличий от контрольной группы, что, скорее всего, связано с длительным течением ЭОП до начала ее лечения и ограничением острой воспалительной фазы заболевания.

IL-8 - самый ранний провоспалительный цитокин, он, так же как и IL-6, повышается в острую воспалительную фазу инфекционных заболеваний, аутоиммунных и иммуновоспалительных заболеваний [32]. IL-8 обладает ангиогенной активностью и играет ключевую роль в образовании новых сосудов. По нашим данным, только у двоих пациентов IL-8 значительно превышал норму 10,9 пг/мл (131 и 150 пг/мл).

Иммуноглобулин IgG является самым распространенным и основным иммуноглобулином сыворотки крови человека, он может составлять до 75\% фракции всех иммуноглобулинов. Концентрация иммуноглобулина подкласса IgG4 в норме ниже, чем других подклассов lgG, и составляет менее 5\% от общего IgG сыворотки крови. Повышение концентрации иммуноглобулинов подкласса lgG4 в сыворотке выявляют при ряде заболеваний, обусловленных инфильтрацией органов плазматическими клетками, секретирующими IgG4 [33, 34]. Последствиями тканевого воспаления являются выраженный фиброз и рубцевание пораженной ткани. В нашем исследовании у $15 \%$ пациентов наблюдалась повышенная концентрация IgG4 (более 1350 мкг/мл) в сыворотке крови, и повышенное содержание IgG4 в IgG (более 5\%) выявлено у 54\% пациентов, что косвенно может свидетельствовать о развитии фиброзных изменений в орбите.

Следует отметить, что у пациентов с некомпенсированным, впервые выявленным тиреотоксикозом при БГ уровни цитокинов и их растворимых рецепторов выявляются в высоких концентрациях [35]. По данным Здор В.В. [36], уровень IL-8 у пациентов с нелеченым тиреотоксикозом

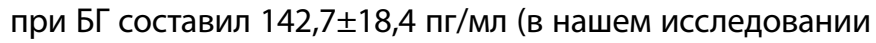
$16,8 \pm 6,4$ пг/мл). В то же время концентрация TGF $\beta 1$ была

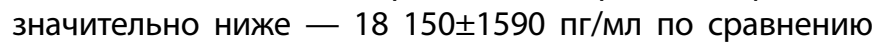
с нашими результатами 24 450,4 1772,2 пг/мл, что свидетельствует о выраженной супрессии регуляторного звена (Treg) у пациентов с некомпенсированным тиреотоксикозом. Впервые гипотеза о дефиците Т-супрессоров была высказана канадским ученым R. Volpe [37], и предположено, что на фоне устранения тиреотоксикоза уровень Т-супрессоров повышается. В нашем исследовании пациенты принимали тиреостатики и поступали в состоянии эутиреоза и субклинического тиреотоксикоза, что отражалось на результатах исследования и снижало влияние тиреотоксикоза на определение цитокинов/рецепторов. Также на результаты исследования могла повлиять продолжительность течения ЭОП до начала ее лечения. В на-

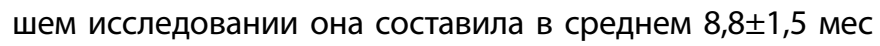
и варьировала от 1 до 48 мес. 


\section{ЗАКЛЮЧЕНИЕ}

Исследованию цитокинов при БГ и ЭОП посвящено много работ. Ограничением многих исследований является то, что высокая концентрация цитокинов была обусловлена некомпенсированным тиреотоксикозом при БГ и в меньшей степени - ЭОП. В нашем исследовании пациенты с БГ получали лечение тиреостатиками и находились в состоянии эутиреоза или субклинического тиреотоксикоза. С другой стороны, пациенты были обследованы не в дебюте ЭОП, а в разные сроки от начала ее манифестации, что могло также повлиять на результаты исследования. У пациентов значимо были повышены солюбилизированные рецепторы «ловушки» цитокинов: sTNFa-R2, sIL-2R и цитокин TGF $\beta 1$, оказывающие супрессивное действие на аутоиммунный процесс за счет активации Т-регуляторных клеток (Т-супрессоров), что косвенно подтверждает гипотезу R. Volpe о повышении регуляторного звена иммунной системы на фоне устранения тиреотоксикоза. sTNFa-R1 как маркер апоптоза коррелировал с активностью ЭОП. С увеличением продолжительности ЭОП концентрация TGF $\beta 1$ как фактора развития фиброза повышалась. О возможном прогрессировании фиброза также могут свидетельствовать высокие концентрации IgG4 в составе IgG.
Таким образом, цитокиновый профиль у пациентов с длительно существующей, нелеченой ЭОП у пациентов с БГ свидетельствует об активации иммунной системы, направленной на супрессию аутоиммунного процесса. Снижение концентрации цитокина TGF $\beta 1$ может свидетельствовать о неблагоприятном течении ЭОП.

\section{ДОПОЛНИТЕЛЬНАЯ ИНФОРМАЦИЯ}

Источник финансирования. Данная работа выполнена при финансовой поддержке Российского научного фонда (грант РНФ №17-75-30035).

Конфликт интересов. Авторы декларируют отсутствие явных и потенциальных конфликтов интересов, связанных с публикацией настоящей статьи

Участие авторов. Свириденко Н.Ю. - эндокринолог: набор материала, проведение исследований, статистический анализ, написание статьи; Бессмертная Е.Г. - офтальмолог: проведение исследований; Беловалова И.М. - эндокринолог: набор материала, рецензия статьи; Михеенков А.А. - эндокринолог: набор материала, проведение исследований; Шеремета М.С. - эндокринолог: набор материала, проведение исследований; Никанкина Л.В. - проведение лабораторных исследований; Малышева Н.М. - проведение лабораторных исследований. Все авторы внесли значимый вклад в проведение исследования и подготовку статьи, прочли и одобрили финальную версию статьи перед публикацией.

\section{СПИСОК ЛИТЕРАТУРЫ | REFERENCES}

1. Wiersinga W, Bartalena L. Epidemiology and prevention of Graves ophthalmopathy. Thyroid. 2002;12(10):855-860.

2. Пантелеева О.Г. Современная концепция механизма развития нарушений зрительных функций при эндокринной офтальмопатии // Автореф. дис. ... д-ра мед. наук. М., 2007. 47 с [Panteleeva OG. The modern concept of the mechanism for development of visual functions disturbances in endocrine ophthalmopathy. [Dissertation] Moscow; 2007.47p.

3. Lee HJ, Li CW, Hammerstad S.S. et al. Immunogenetics of autoimmune thyroid diseases: a comprehensive review. J. Autoimmun. 2015;64:82-90. doi: 10.1016/j.jaut.2015.07.009

4. Krigger CC, Place RF, Bevilacqua C, et. al. TSH/IGF-1 receptor cross talk in Graves' ophthalmopathy pathogenesis. J Clin Endocrinol Metab. 2016;101:2340-2347. doi: 10.1089/thy.2017.0105

5. Rotondi M, Carbone A, Coperchini F, et. al. Diagnosis of endocrine disease: IgG4-related Thyroid Autoimmune Disease. Eur J Endocrinol. 2019;180(5):175-183. doi: 10.1530/EJE-18-1024

6. Salvi M, Campi I. Medical Treatment of Graves' Orbitopathy. Horm Metab Res. 2015:47:1-10. doi: 10.1055/s-0035-1554721

7. Gorman C, Garrity J, Fatourech V, Bahn R. A prospective, randomized double-blind, placebo-controlled study of orbital radiotherapy for Graves' ophthalmopathy. Ophthalmology. 2020;127(4):160-171. doi: 10.1016/S0161-6420(01)00632-7

8. Paridaens D, van den Bosch WA, Krenning E. The effect of etanercept on Graves' ophthalmopathy: a pilot study. Eye. 2005;19:1286-1289.

9. Durrani OM, Reuser TQ, Murray PI. Infliximab: a novel treatment for sightthreatening thyroid associated ophthalmopathy. Orbit. 2005;24:117-119.

10. Douglas R, Kahaly G, Patel A. et al. Teprotumumab for the Treatment of Active Thyroid Eye Disease. N Engl J Med. 2020;382(4):341-352.

11. Kahaly G, Bartalena L, Hegedüs L, et al. 2018 European Thyroid Association Guideline for the Management of Graves' Hyperthyroidism. Eur Thyroid J. 2018;7:167-186. doi: 10.1159/000490384

12. Bartalena L, Baldeschi L, Boboridis K, et al. European Thyroid Association/European Group on Graves' Orbitopathy Guidelines for te Management of Graves' Orbitopathy. Eur Thyroid J. 2016;5:9-26. doi: 10.1159/000443828

13. Wiersinga WM, Perros P, Kahaly GJ, et al. Clinical assessment of patients with Graves' Orbitopathy: The European Group on Graves' Orbitopathy (EUGOGO) recommendations to generalists, specialists and clinical researchers. Eur J Endocrinol. 2006;155:387-389.
14. Дедов И.И., Мельниченко Г.А., Свириденко Н.Ю., и др. Федеральные клинические рекомендации по диагностике и лечению эндокринной офтальмопатии при аутоиммунной патологии щитовидной железы // Проблемы эндокринологии. - 2015. T. 61. - №1. - C. 61-74. [Dedov II, Melnichenko GA, Sviridenko NY, et al. Federal clinical recommendations on diagnostics and treatment of endocrine ophthalmopathy associated with autoimmune thyroid pathology. Problems of endocrinology. 2015;61(1):61-74. (In Russ.)]. doi: https://doi.org/10.14341/probl201561161-74.

15. Чепурина А.А., Свириденко Н.Ю., Ремизов О.В., Беловалова И.М. Визуализирующие методы исследования в диагностике эндокринной офтальмопатии// Медицинская визуализация. 2012. - №1. - C. 36-44. [Chepurina AA, Sviridenko NY, Remizov OV, Belovalova IM. Imaging Methods in the Diagnosis of ThyroidAssociated Orbitopathy. Medical Visualization. 2012;1: 36-44. (In Russ.)].

16. Smith TJ, Hegedus L. Graves' disease. N Engl J Med. 2016;375:1552-1565. doi: 10.1056/ NEJMra1510030

17. Свириденко Н.Ю., Лихванцева В.Г., Беловалова И.М., Шеремета М.С., Табеева К.И. Антитела к рецептору ТТГ как предикторы тяжести и исходов эндокринной офтальмопатии у пациентов с болезнью Грейвса // Проблемы эндокринологии. — 2011. — №2. - С. 23-26. [Sviridenko NYu, Likhantseva VG, Belovalova IM, et al. Anti-TSH receptor antibodies as predictors of the severity and outcome of endocrine ophthalmopathy in the patients presenting with Graves' disease. Problems of endocrinology. 2011;57(2):23-26. (In Russ.)]. doi: https://doi.org/10.14341/probl201157223-26.

18. Bahn R. Graves' Ophthalmopathy. N Engl J Med. 2010;362:726-738. doi: 10.1056/NEJMra0905750

19. Ярилин А.А. Иммунология. - М.: ГЭОТАР-Медиа, 2010. C. 190-226. [Yarilin AA. Immunologia. M.: GEOTAR-Media, 2010. P. 190-226. (In Russ.)]

20. Стагниева И.В., Бойко Н.В., Гукасян Е.Л., Бачурина А.С. Цитокины в диагностике воспалительных заболеваний верхних дыхательных путей // Российская ринология. — 2017. - Т. 25. - №4. - C. 43-47. [Stagnieva IV, Boiko NV, Gukasyan EL, Bachurina AS.The role of cytokines in the diagnostics of inflammatory diseases of the upper respiratory tract. Russian Rhinology=Rossiyskaya Rinologiva. 2017;25(4):43-47 (In Russ.)]. doi:10.17116/ROSRINO201725443-47 
21. Симбирцев А.С. Цитокины в патогенезе и лечении заболеваний человека. - СПб: Фолиант, 2018. - C. 10-55 [Simbirtsev AS. Cytokines in the pathogenesis and treatment of human diseases. SPb: Foliant, 2018. P. 10-55 (In Russ.)].

22. Rajaii F, McCoy AN, Smith TJ. Cytokines are both villains and potential therapeutic targets in thyroid-associated ophthalmopathy: From bench to bedside. Expert Rev Ophthalmol. 2014;9(3):227-34.

23. Myśliwiec J, Kretowsk A, Stepień A, Kinalska I. Serum Levels of Soluble TNFa Receptors (sTNFR1 and sTNFR2) during Corticosteroid Treatment in Patients with Graves' Ophthalmopathy. Immunological investigations. 2004;33(1):61-68. doi: $10.1081 /$ IMM-120027685

24. Симбирцев А.С. Цитокины в патогенезе инфекционных и неинфекционных заболеваний человека // Медицинский академический журнал. - 2013. - Т. 1. - №3. - С.18-41. [Simbirtsev AS. Cytokines in the pathogenesis of infectious and noninfectious human diseases. Medical academic journal. 2013; 1 (3):18-41 (In Russ.)]. doi: https://doi.org/10.17816/MAJ13318-41

25. Faustman D, Davis M. TNF Receptor 2 and Disease: Autoimmunity and Regenerative Medicine. Frontiers in Immunology. 2013;4:478. doi: 10.3389/fimmu.2013.00478

26. Таскина Е.С., Харинцева С.В. Морфофункциональная характеристика и иммунологическая регуляция функции орбитальных фибробластов при эндокринной офтальмопатии // Клиническая и экспериментальная тиреоидология. - 2018. - Т. 14. - №4. - С. 183-191. [Taskina ES, Kharintseva SV. Morphofunctional characteristics and immunological regulation of the orbital fibroblasts function in endocrine ophthalmopathy. Clinical and experimental thyroidology. 2018;14(4):183-191]. doi: https://DOl.org/10.14341/ket10147

27. Kajdaniuk D, Marek B, Niedziołka-Zielonka D, Foltyn W, Nowak M, Siemińska L, Borgiel-Marek H, Głogowska-Szeląg J, Ostrowska Z, Drożdż L, Kos-Kudła B. Transforming growth factor $\beta 1$ (TGF $\beta 1$ ) and vascular endothelial growth factor (VEGF) in the blood of healthy people and patients with Graves' orbitopathy — a new mechanism of glucocorticoids action? Endocrinol Pol. 2014;65(5):348-56. doi: 10.5603/EP.2014.0048.
28. Khalilzadeh O, Noshad S, Rashidi A, Amirzargar A. Graves Ophthalmopathy: A Review of Immunogenetics. Current Genomics. 2011:12:564-575.

29. Pandiyan P, Zhu J. Origin and functions of pro-inflammatory cytokine producing Foxp3+ regulatory T cells. Cytokine. 2015;76 (1):13-24 doi: 10.1016/j.cyto.2015.07.005

30. Slowik M, Urbaniak-Kujda D, Bohdanowicz-Pawlak A Kapelko-Slowik K, Dybko J, Wolowiec D, Jazwiec B, Daroszewski J. CD8+CD28-lymphocytes in peripheral blood and serum concentrations of soluble interleukin 6 receptor are increased in patients with Graves' orbitopathyand correlate with disease activity. Endocr Res. 2012;37(2):89-95. doi:10.3109/07435800.2011.635622.

31. Ruggeri RM, Barresi G, Sciacchitano S, et al. Immunoexpression of the CD30 ligand/CD30 and IL-6/IL-6R signals in thyroid autoimmune diseases. Histol Histopathol. 2006;21(3):249-256. doi: 10.14670/HH-21.249

32. Arican $O$, Aral $M$, Sasmaz $S$, Ciragi P. Serum levels of TNF-a, IFN- $\gamma$, IL-6, IL-8, $\mathrm{IL}-12, \mathrm{IL}-17$, and IL-18 in patients with active psoriasis and correlation with disease severity. Mediators of Inflammation. 2005;5:273-279.

33. Yu SH, Kang JG, Kim CS et al. Clinical Implications of Immunoglobulin G4 to Graves' Ophthalmopathy. Thyroid. 2017; 27(9):1185-1193. doi: 10.1089/THY.2017.0126

34. Bozkirli E, Bakiner OS, Ersozlu Bozkirli ED, et al. Serum Immunoglobulin G4 levels are elevated in patients with Graves ophthalmopathy. Clin Endocrinol (Oxf). 2015;83(6):962-967. doi: 10.1111/CEN.12671

35. Ganesh BB, Bhattacharya P, Gopisetty A, Prabhakar BS. Role of Cytokines in the Pathogenesis and Suppression of Thyroid Autoimmunity. J. Interferon Cytokine Res. 2011;31:721-731. doi: 10.1089/jir.2011.0049

36. Здор В.В., Маркелова Е.В. Патогенетическая роль системы цитокинов при аутоиммунном тиреотоксикозе // Клиническая и экспериментальная тиреоидология. - 2013. - Т. 9. - №4. - С. 27-30. [Zdor W, Markelova EV. The State and Interactions of the System of Cytokines in Autoimmune Thyrotoxicosis. Clinical and experimental thyroidology. 2013;9(4):27-30 (In Russ.)].

37. Volpé R. Immunological aspects of thyroid disease. Triangle. 1984:23:95-109.

Рукопись получена: 15.08.2020. Одобрена к публикации: 01.10.2020. Опубликована online: 14.12.2020.

ИНФОРМАЦИЯ ОБ АВТОРАХ [AUTHORS INFO]

* Свириденко Наталья Юрьевна, д.м.н., професcop [Natalya Yu. Sviridenko, MD, PhD, Professor]; адрес: Россия, 117036, Москва, ул. Дм. Ульянова, д. 11 [address: 11 Dm. Ulyanova street, 117036 Moscow, Russia]; ORCID: http://orcid.org/0000-0002-8538-5354; eLibrary SPIN: 5889-6484; e-mail: natsvir@inbox.ru

Бессмертная Елена Григорьевна, к.M.н. [Elena G. Bessmertnaya, PhD]; ORCID: http://orcid.org/0000-0001-5910-6502; eLibrary SPIN: 1273-3426; e-mail: bessmertnaya.eg@gmail.com

Беловалова Ирина Михайловна, к.м.н. [Irina M. Belovalova, PhD]; ORCID: http://orcid.org/0000-0002-9954-7641; eLibrary SPIN: 7158-0658; e-mail: belovalova.irina@endocrincentr.ru

Михеенков Александр Александрович [Alexander A. Mikheenkov]; ORCID: http://orcid.org/0000-0001-9981-1767; eLibrary SPIN: 6824-5971; e-mail: Mikheenkov_Alexander@mail.ru

Шеремета Марина Сергеевна, к.м.н. [Marina S. Sheremeta, MD, PhD]; ORCID:

http://ORCID.org/0000-0003-3785-0335; eLibrary SPIN: 7845-2194

Никанкина Лариса Вячеславовна, к.M.н. [Nikankina Larisa V. PhD]; ORCID: http://orcid.org/0000-0001-8380-3825; eLibrary SPIN: 2794-0008

Малышева Наталья Михайловна, к.б.н. [Natalia M. Malysheva, PhD]; ORCID: https://orcid.org/0000-0001-7321-9052; eLibrary SPIN: 5793-2550

\section{ЦИТИРОВАТЬ:}

Свириденко Н.Ю., Бессмертная Е.Г., Беловалова И.М., Михеенков А.А., Шеремета М.С., Никанкина Л.В., Малышева Н.М. Аутоантитела, иммуноглобулины и цитокиновый профиль у пациентов с болезнью Грейвса и эндокринной офтальмопатией// Проблемы эндокринологии. - 2020. — Т. 66. — №5. - C. 15-23. doi: https://doi.org/10.14341/probl12544

\section{TO CITE THIS ARTICLE:}

Sviridenko NY, Bessmertnaya EG, Belovalova IM, Mikheenkov AA, Sheremeta MS, Nikankina LV, Malysheva NM. Autoantibodies, immunoglobulins and cytokine profile in patients with graves' disease and graves' orbitopathy. Problems of Endocrinology. 2020;66(5):15-23. doi: https://doi.org/10.14341/probl12544 